\title{
Espaces publics et environnement dans les politiques urbaines à Paris et à Berlin
}

\author{
Antoine Fleury \\ UMR 8504 Géographie-cités \\ 13, rue du Four \\ 75006 PARIS \\ afleury@parisgeo.cnrs.fr
}

\begin{abstract}
Résumé
Depuis les années 1970, même si c'est à des rythmes différents selon les métropoles européennes, les pouvoirs publics ont progressivement fait de l'espace public un outil pour améliorer l'environnement urbain. Aujourd'hui, l'aménagement des espaces publics occupe une place importante dans les politiques urbaines, à la fois pour changer les modes de déplacement ou pour revaloriser le cadre de vie dans la proximité. Ce choix politique va de pair avec l'élaboration de nouveaux modes de production centrés d'une part sur la concertation avec les acteurs locaux et les habitants, dont les préoccupations environnementales sont importantes, et d'autre part sur la coordination des différents acteurs institutionnels, parmi lesquels les acteurs en charge de l'environnement se sont renforcés. La mise en regard de Paris et de Berlin montre combien cette double tendance revêt un caractère profondément européen, même si les espaces publics occupent une place variable d'une ville à l'autre et d'un pays à l'autre. Dans tous les cas, la place croissante des enjeux environnementaux dans ces politiques publiques peut néanmoins entrer en tension avec les autres dimensions de la ville et de ses espaces publics.
\end{abstract}

Mots-clés

Espaces publics, ville, environnement, déplacements, cadre de vie, Paris, Berlin

\section{Abstract}

\section{Public spaces and the environment in the urban policies of Paris and Berlin}

Since the 1970s, public spaces have increasingly become the instruments of urban environment improvement in European metropolis. Urban policies are currently mainly devoted to upgrading public spaces, either by modifying modes of transportation or by renewing the living environment. New modes of public action involve both local stakeholders and residents for whom these environmental issues are of great importance. But public authorities also aim for better coordination of institutional actors, especially those in charge of environmental matters. Confronting the case studies of Paris and Berlin, we demonstrate here the extent to which this trend may be qualified as truly European in 
nature, despite local differences. This environmental issues and the resulting public policies may sometimes conflict with other features of cities and their public spaces.

Key words

Public spaces, city, environment, transportation, living environment, Paris, Berlin

\section{Zusammenfassung}

\section{Umwelt und öffentliche Räume innerhalb der Stadtpolitiken in Paris und Berlin}

Die öffentliche Räume sind zentrale Aspekte öffentlicher Politiken zur Verbesserung des städtischen Umfeldes geworden. Diese und ähnliche Entwicklungen sind im Kontext wichtiger politischer und kultureller Veränderungen erfolgt. Seit den 1970er Jahren sind neue Akteure ins Kräftefeld städtebaulicher Planung getreten, darunter Einwohnervereine und grüne Aktivisten, die die Repräsentationen, Praktiken und das Management im Bereich Städtebau grundlegend verändert haben. Heutzutage bedienen sich Stadtentwickler immer häufiger der Neugestaltung öffentlicher Räume: durch das Bevorzugen von Fußgängern, Radfahrern und öffentlichen Verkehrsmitteln einerseits; durch die Verbesserung des Wohnumfeldes andererseits (in Bezug auf die Sicherheit von Fußgängern, die Qualität des städtebaulichen Materials und die Vermehrung von Grünflächen). Die Gestaltung öffentlicher Räume liegt nicht mehr wie früher in den Händen der Stadtverwaltung und der Experten allein, sondern erfolgt nun im Einvernehmen mit lokalen Akteuren und Einwohnern, die sich gegenüber den öffentlichen Behörden immer mehr behaupten. Außerdem wurde die Verwaltung selbst dadurch ebenfalls neu strukturiert im Sinne einer größeren Demokratisierung und Berücksichtigung von Umweltfragen. So wurden institutionelle Ebenen und technische Dienste miteinander verknüpft und neue Akteure mit erhöhten Umweltkompetenzen eingeschaltet, wie z. B. Architekten und Landschaftsplaner.

Der Vergleich zwischen Paris und Berlin lässt auf einen europaweiten und zeitgleichen, aber stark differenzierten Prozess schließen. In Paris wurden Umweltfragen nur zögernd mit einbezogen, während sie in (West-)Berlin zu den ersten Maßnahmen gehörten. Nun ist die Lage beinahe umgekehrt. In Paris hat sich die Suche nach Alternativen zum Kraftfahrzeug und die Neu-Gestaltung öffentlicher Räume mit der Schaffung von „espaces civilisés" und "quartiers verts" intensiviert. Im Zuge der Wiedervereinigung zeigte sich in Berlin erneut, wie akut umweltpolitische Fragen waren, u. a. durch die Zunahme des motorisierten Verkehrs. Das „westliche“ Modell der 1980er Jahre wurde auf die östlichen Berliner Bezirke ausgedehnt. Doch in Berlin ist die Neugestaltung öffentlicher Räume nunmehr im Kontext finanzieller Engpässe nicht mit dem zu vergleichen, was in Paris geschieht. Die Maßnahmen sind pragmatischer und beschränken sich auf die (u. a. östlichen) sozial benachteiligten Viertel. In beiden Städten jedoch stehen die erhöhten Umweltforderungen öffentlicher Politiken im Widerspruch zu den anderen Dimensionen der Stadt. So lassen sich öffentliche Räume weder zu bloßen Verkehrszwecken instrumentalisieren, die oftmals zu einer longitudinalen Fraktionierung der Straßen führen, noch auf ihre Funktion als Wohnumfeld für Einwohner reduzieren, was etliche Kategorien von Benützern ausschließt.

\section{Stichwörter}

Öffentliche Räume, Stadt, Umwelt, Verkehr, Wohnumfeld, Paris, Berlin 
Le terme d'espace public est utilisé en urbanisme depuis les années 1970. II renvoie à des formes urbaines - rues, places ou boulevards - tout en faisant référence à la notion d'espace public, forgée en philosophie politique pendant la décennie précédente (Tomas, 2002). L'objectif est donc clairement affiché : l'espace public est un instrument pour recréer du lien social, réactiver une urbanité perçue comme en crise. II s'agit désormais de réinventer des espaces publics où règne une certaine mixité, où des gens différents puissent se côtoyer. Dans les années 1990, le terme se diffuse largement en Europe, devenant une "quasi-doctrine en aménagement " (Toussaint \& Zimmermann, 2001). Au même moment, les enjeux environnementaux sont progressivement intégrés dans les politiques urbaines. Les espaces publics sont alors de plus en plus associés à l'environnement (Haubold, 1997), ce qui se comprend aisément: dans la ville dense, ils supportent la majorité des déplacements, et notamment la circulation automobile, considérée comme une source non négligeable de pollution atmosphérique. A cette question de santé publique s'ajoutent les attentes croissantes en termes d'amélioration du cadre de vie.

Paris et Berlin sont deux capitales politiques marquées par l'histoire, qui entretiennent entre elles des liens étroits depuis longtemps ${ }^{1}$. Elles appartiennent toutes deux à un système des villes européennes en construction, et l'action publique y est traditionnellement forte. Pour autant, ces deux métropoles sont très différentes du point de vue de la taille et des densités (carte $n^{\circ} 1$ ), des systèmes politiques ${ }^{2}$ ou encore des mentalités, avec notamment une fibre environnementale plus ancrée à Berlin. Elles présentent de surcroît des contextes économiques et sociaux contrastés. Au-delà de ces différences, une série d'entretiens menés depuis 2004 avec des acteurs institutionnels, des urbanistes et des architectes, des militants associatifs et des acteurs locaux, ainsi que l'analyse qualitative d'un large corpus de documents ${ }^{3}$ nous permettent d'affirmer que non seulement les pouvoirs publics ont fait de l'espace public un outil pour améliorer l'environnement des espaces centraux ${ }^{4}$, mais que cette dimension de l'espace public prend aussi une place croissante, ce qui pose selon nous un certain nombre de problèmes par rapport au sens de la ville et de ses espaces publics.

En se situant au croisement des expériences parisiennes et berlinoises ${ }^{5}$, cet article se propose de réfléchir au rôle des enjeux environnementaux dans l'action publique sur les espaces publics aujourd'hui - essentiellement les rues et les places - et à la manière dont la prise en compte de ces enjeux s'articule aux autres dimensions de l'espace public, du point de vue des lieux et de la ville comme du point de vue des acteurs en jeu. Pour cela, il conviendra tout d'abord de se demander comment l'espace public est devenu un outil pour

\footnotetext{
${ }^{1}$ Pour un cadrage sur la comparaison Paris/Berlin, voir Duhem, Grésillon \& Kohler, 2000.

${ }^{2}$ Berlin est une ville-Etat au sein d'un régime fédéral, alors que la ville de Paris a mis du temps à bénéficier de la décentralisation. Voir notamment Rytlewski, 1999 et Spitz, 2004.

${ }^{3}$ Documents officiels, études, articles de presse, sites Internet, etc.

${ }^{4}$ Nous nous limiterons ici aux espaces centraux de ces deux métropoles: Paris intra-muros et Berlin Innenstadt (ville intérieure) dont la surface est d'ailleurs largement comparable et qui constituent les parties les plus denses de ces métropoles.

${ }^{5}$ La méthode du croisement, formalisée par M. Werner et B. Zimmermann (2004), se propose de dépasser la comparaison d'une part et l'étude des transferts culturels d'autre part. Les objets ne sont pas seulement considérés «les uns par rapport aux autres, mais également les uns à travers les autres, en termes de relations, d'intersections... » (p. 22).
} 
améliorer l'environnement urbain et ensuite d'évaluer la place de ce dernier dans les politiques mises en œuvre ; à partir de là, il sera possible d'identifier les acteurs concernés, au sein de la puissance publique comme de la société civile, et de mesurer combien la prise en compte des enjeux environnementaux a pu contribuer aux recompositions intervenues dans les modes d'organisation et dans les relations qui s'établissent entre ces acteurs; enfin, nous tenterons de poser un regard critique sur ces politiques et sur les jeux d'acteurs qui les sous-tendent.

Carte $n^{\circ}$ 1. L'espace bâti et les découpages administratifs de Paris et Berlin

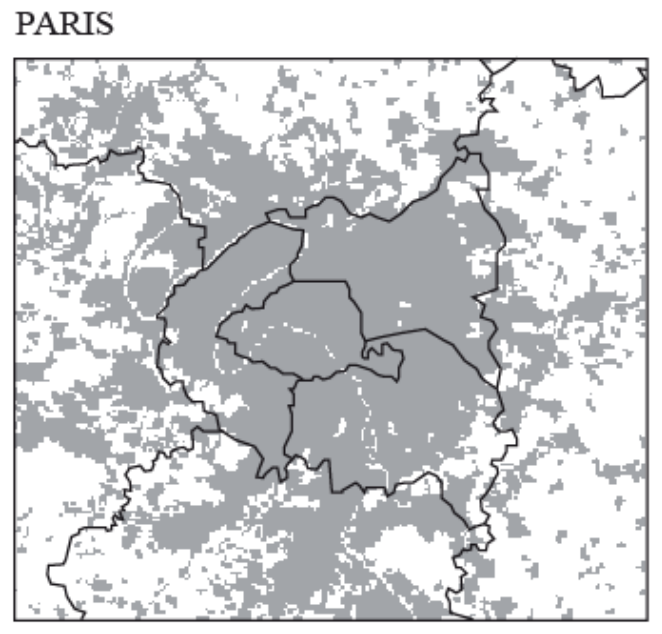

Espace bâti

Limites administratives
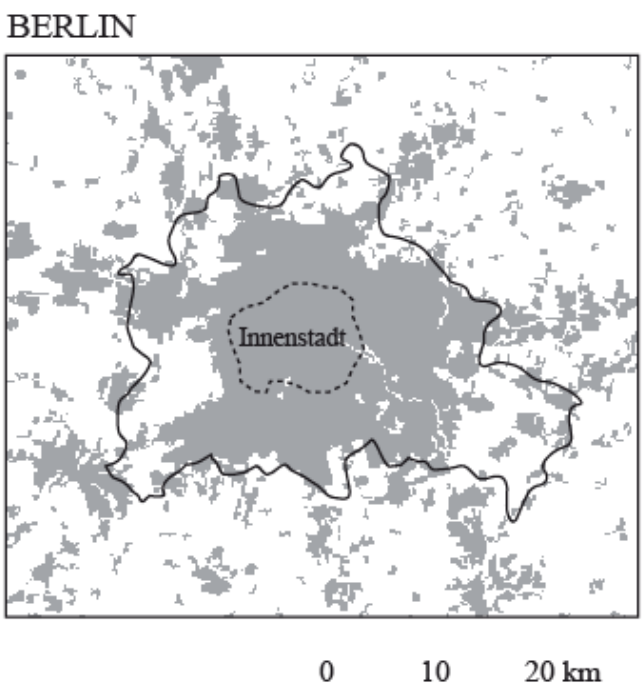

Sources : European Environment Agency, Joint Research Center Conception : Marianne Guérois et Antoine Fleury

\section{Les espaces publics au cœur des préoccupations environnementales depuis les années 1970}

Les politiques actuelles se sont progressivement construites depuis les années 1970. On ne saurait donc évoquer ces politiques avant de rappeler en quoi elles sont en rupture avec des décennies d'aménagement des espaces publics peu soucieuses de l'environnement urbain.

Durant les Trente Glorieuses, l'objectif des pouvoirs publics était en de régler la ville sur l'automobile, dans une optique presque exclusivement fonctionnelle. Les espaces publics hérités du XIX ${ }^{\mathrm{e}}$ siècle sont alors bouleversés dans leurs formes comme dans leurs usages ; ils laissent parfois place à des voies express qui destructurent le tissu urbain hérité. A Paris, les chaussées des boulevards hausmmaniens sont élargies aux dépens des trottoirs et des plantations d'alignement; les places sont vouées au stationnement (Flonneau, 2001) ; une voie express est même aménagée sur les berges de la Seine, préfurant tout un réseau au cœur du tissu ancien. Cette transformation est plus importante encore à Berlin, où les destructions de la guerre ont permis de tracer de larges autoroutes urbaines, aussi bien à Berlin-Ouest qu'à Berlin-Est (Bodenschatz, 1987). 
C'est la vague contestataire des années 1960 et 1970 qui conduit, en Europe occidentale, à un revirement des pouvoirs publics (Decroly et al., 2003). A Paris comme à Berlin, des " luttes urbaines " se multiplient, remettant en cause des choix d'aménagement accusés de dégrader l'environnement urbain (Cherki \& Mehl, 1979, Bodenschatz, 1987). Dans un premier temps, le mouvement conduit les pouvoirs publics à abandonner la construction de certaines infrastructures ${ }^{6}$ ainsi que la politique de rénovation urbaine au profit d'une réhabilitation de la ville héritée. Dans un second temps sont développées des politiques en faveur du cadre de vie (Joye et al., 1994). Au cours de ce processus, de nouveaux acteurs ont émergé. Parmi eux, les habitants sont remis sur le devant de la scène, même si ce sont les classes moyennes et supérieures - dont la présence tend déjà à se renforcer dans les centres urbains à cette époque ${ }^{7}$ - qui sont particulièrement concernées. Intervenant sous la forme d'initiatives citoyennes ou dans le cadre d'associations, ces habitants revendiquent de manière récurrente la préservation de leur cadre de vie. En Allemagne de l'Ouest et plus tard en France, les partis verts se sont également imposés dans le débat politique, et avec eux la question environnementale au sens large. Quant aux pouvoirs publics, ils travaillent avec un éventail plus large de professionnels de la ville, comprenant de plus en plus d'architectes et de paysagistes, considérés comme plus sensibles que les ingénieurs aux préoccupations des habitants.

Aménager pour restreindre la place de l'automobile: Berlin-Ouest et les Expositions Internationales d'Architecture

Bien évidemment, l'évolution des politiques publiques s'est faite à des rythmes différents selon les villes. Les villes ouest-allemandes ont été plus tôt concernées que les villes françaises. Elle s'inscrivent en cela dans le vaste mouvement qui touche les villes d'Europe du Nord depuis les années 1960, à partir des Pays-Bas où se développe précocement le concept des "cours urbaines"(Olagnier, 2003). Parmi ces villes, BerlinOuest ${ }^{8}$ occupe cependant une place singulière. Dans un contexte de Guerre froide, la ville apparaît en effet comme une vitrine du monde libre. Au moment où se développent les "luttes urbaines", elle a donc dû prendre en compte au plus vite les exigences d'une population dont toute une frange, très ancrée à gauche, est très sensible aux valeurs environnementales et à la préservation de son cadre de vie. Le processus s'y déploie donc assez rapidement à partir de la fin des années 1970. II associe aux acteurs publics les habitants et les architectes dans le cadre de concours, notamment en amont des Internationale Bau Ausstellungen (Expositions Internationales d'Architecture). Une réflexion s'engage alors sur la forme à donner aux espaces publics; ceux-ci se retrouvent au cœur de l'action publique, en relation avec la nouvelle politique de réhabilitation urbaine. Tout l'enjeu est de redonner de la qualité à ces espaces. Ainsi, une grande partie des espaces publics est repensée, avec la généralisation du principe de Verkehrsberuhigung

\footnotetext{
${ }^{6}$ On peut citer la radiale Vercingétorix ou la voie Paris-Rive-Gauche à Paris (Flonneau, 2001) ou la voie rapide Est-Ouest à Berlin-Kreuzberg (Bodenschatz, 1987).

${ }^{7} \mathrm{Cf}$. les travaux pionniers d'H. Coing (1966) puis les premières recherches portant sur ce que l'on n'appelait pas encore la gentrification (Chalvon-Demersay, 1984).

${ }^{8}$ De l'autre côté du mur, le régime communiste a mis en place une politique d'inspiration fonctionnaliste en construisant des grands axes de transit pour les voitures, reléguant les autres modes de déplacements dans les zones réservées ou les voies de desserte locale. Pour autant, la circulation n'est alors pas un problème vu le faible nombre d'automobilistes.
} 
(apaisement du trafic) et le développement des pistes cyclables aussi bien sur les grands axes que dans les secteurs résidentiels (figure $n^{\circ} 1$ ). En parallèle, la présence végétale est renforcée, avec la plantation d'arbres et l'installation de jardinières dans les rues et sur les places dégagées de la circulation automobile et d'une partie du stationnement (Schöbel, 2003). Les Expositions Internationales d'Architecture font alors de Berlin un modèle sur le plan urbanistique, y compris dans l'aménagement de ses espaces publics (Schlusche, 1997).

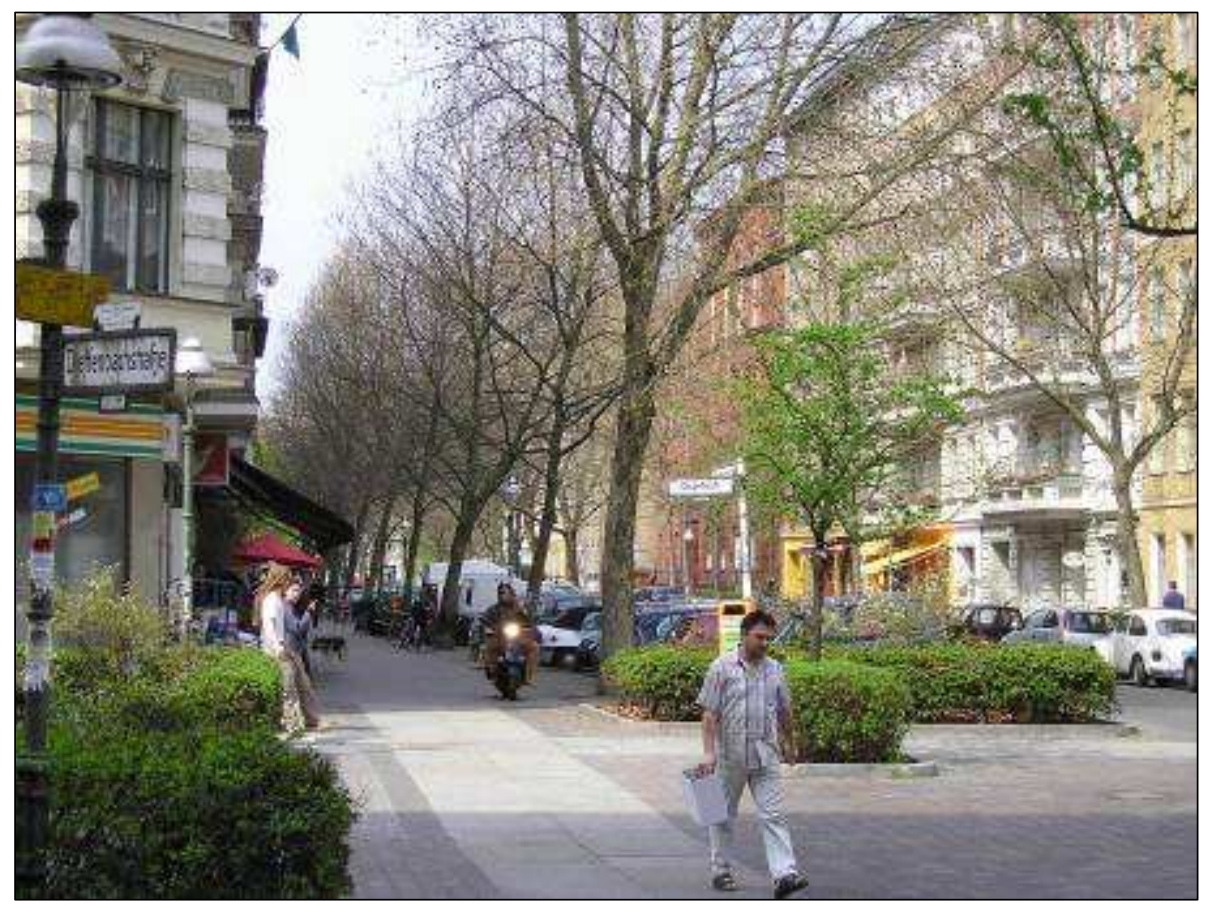

Figure $n^{\circ} 1$. Une "verkehrsberuhigte Zone » à Berlin-Kreuzberg (回 Antoine Fleury, 26-04-2006)

\section{Aménager pour réguler la circulation et protéger les piétons: l'esprit du " plan de circulation » à Paris}

La situation est assez différente à Paris, la capitale française continuant à être gérée selon un régime d'exception qui donne une place considérable à l'Etat. Malgré la réforme institutionnelle de 1975, le Maire élu en 1977 poursuit la politique engagée par l'Etat à la suite des "luttes urbaines » et du revirement accompli par V. Giscard d'Estaing en 1974. Malgré l'abandon des grands projets d'infrastructure, et ce jusqu'au milieu des années 1990, l'action de la Mairie vise à fluidifier la circulation et à faciliter le stationnement. L'enjeu est toujours de "retoucher l'espace public pour améliorer la circulation »". Aussi favorable à la circulation automobile soit-elle, cette politique tend néanmoins à intégrer la protection du patrimoine et l'amélioration de la sécurité des piétons - en " recalibrant " les rues et en développant le " mobilier de défense " (potelets, barrières, etc.) - bref à mieux prendre en compte l'environnement urbain dans sa dimension locale. Certains espaces publics de quartier sont réaménagés, mais seulement de manière ponctuelle, à l'occasion par exemple de la construction d'un parc de stationnement souterrain ou du

\footnotetext{
${ }^{9}$ Extrait de l'entretien avec P. Hattenberger, 01/09/2005.
} 
" recalibrage " d'une rue. Ce sont en fait moins les espaces publics de proximité que les places et avenues prestigieuses - comme les Champs Elysées ou la place de l'Hôtel de Ville - qui sont requalifiées, l'enjeu environnemental passant ici au second plan derrière le marketing urbain.

Dans les années 1990, les services de la Ville s'engagent cependant dans une réflexion plus globale sur les espaces publics. L'objectif est de rendre plus cohérents les aménagements déjà engagés, comme en témoigne le Guide de l'Espace Public rédigé à cette époque. II faut noter que les architectes-voyers ${ }^{10}$ jouent un rôle important dans cette réflexion. A la demande des élus locaux, sensibles aux exigences de leurs administrés, la municipalité élue en 1995 s'engage également dans une politique plus favorable aux " circulations douces » et à la qualité de vie. C'est un tournant dans la politique parisienne, qui rejoint alors le mouvement engagé plusieurs décennies auparavant en Europe du Nord. Dans les années qui suivent sont aménagés les " quartiers tranquilles » et mis en place les premiers jalons d'un réseau cyclable. A la différence de Berlin, les acteurs locaux sont cependant peu associés lors de ces programmes d'aménagement, hormis pour la mise en place du réseau cyclable, marquée par une forte implication des associations. Ces mesures n'en sont pas moins importantes puisque la politique menée actuellement ne fait finalement que reprendre en l'amplifiant cette action ${ }^{11}$.

\section{Aménager les espaces publics pour améliorer l'environnement urbain aujourd'hui}

L'aménagement des espaces publics constitue aujourd'hui un outil pour améliorer l'environnement urbain, quelle que soit l'échelle (Haubold, 1997). D'une métropole à l'autre, il n'est cependant mobilisé ni de la même manière, ni avec la même intensité. Ainsi, alors que Paris donne la priorité à l'aménagement des espaces publics, Berlin met en œuvre une politique beaucoup plus pragmatique où les espaces publics ne sont pas au premier plan.

\section{Un outil plus ou moins central dans la politique des déplacements}

La protection de l'environnement (Umwelt) - au sens du milieu dans lequel la société évolue - est aujourd'hui centrale dans l'action publique. II s'agit de limiter la pollution, de réduire la consommation énergétique ou encore de retraiter les déchets. Or, dans les grandes métropoles, l'automobile est considérée comme l'un des principaux facteurs de pollutions, qu'elles soient atmosphériques ou sonores. C'est pourquoi, dans un objectif de santé publique, ce mode de déplacement est désormais perçu comme inadapté à la ville dense (Dupuy, 1995). Des politiques plus favorables aux transports en commun et aux "circulations douces" (bicyclette, roller) sont mises en œuvre, pour lesquelles l'aménagement des espaces publics constitue l'un des outils possibles (Bourlon \& Villot,

\footnotetext{
${ }^{10}$ Créés par une délibération du Conseil de Paris (08/07/1991), les architectes-voyers exercent « des fonctions techniques ou administratives dans les domaines de l'architecture, de l'urbanisme, de la construction " et assurent diverses missions comprenant notamment la programmation, la conception ou la maîtrise d'œuvre.

${ }^{11}$ Pour les liens entre la politique de J. Tibéri (1996 et 2001) et celle de B. Delanoë (élu en 2001), voir Fleury, 2007.
} 
2004 ; SenStadt, 2005). Cependant, si l'aménagement des espaces publics joue un rôle dans les politiques des déplacements, c'est à des degrés divers selon les villes.

A Paris, la municipalité élue en $2001^{12}$ met en place une politique des déplacements ambitieuse. Son action s'appuie sur un constat : seulement $6 \%$ des espaces publics sont affectés aux piétons, aux transports en commun et aux " circulations douces ", alors qu'ils représentent plus de $80 \%$ des déplacements dans Paris (DREIF, 2004). La Mairie s'est donc fixé comme objectif de réduire la place de l'automobile au profit de ces autres modes de déplacement ${ }^{13}$. Pour cela, contrairement à Londres qui a mis en place un péage urbain ou Rome qui a établi un permis spécial pour entrer dans le centro storico, ce sont les réaménagements de la voirie qui sont privilégiés. Cela s'explique en partie par une tradition très ancrée d'aménagement des espaces publics depuis Haussmann, incarnée par la Direction de la voirie (Landau, 1993), et par des marges de manœuvre financières qui demeurent considérables. La politique mise en œuvre s'appuie sur un ensemble de " grands projets " où s'impose un nouveau partage de la voirie. II s'agit tout d'abord des " quartiers verts ", qui visent, selon le principe des "zones 30 ", à diminuer la vitesse et à réduire le transit automobile au profit des "circulations douces". Ils consistent principalement en une réorganisation du plan de circulation et un réaménagement des carrefours. Les " espaces civilisés » concernent quant à eux les grands axes de circulation : élargissement des trottoirs, aménagements spécifiques pour les autobus et les vélos, réduction du nombre de files de circulation (figure 2). Sur ces axes se greffent enfin des aménagements en réseaux, à l'échelle de la ville. Il s'agit d'une part du programme "Mobilien », qui a pour objectif d'améliorer les transports en commun de surface en termes de régularité et de rapidité ${ }^{14}$, et dont la mise en place se traduit par la construction de sites propres pour les lignes de bus les plus fréquentées. II s'agit d'autre part de l'aménagement d'un réseau de pistes cyclables, dont la cohérence est assurée par un schéma directeur à l'échelle de la capitale.

Depuis la réunification, le problème de la circulation automobile se pose de nouveau de manière aiguë à Berlin. La situation s'explique par un étalement urbain important et par le rattrapage du taux de motorisation dans la partie est de la ville (Häußermann \& Kapphan, 2002). Le principe de réduction de la circulation s'inscrit donc au cœur de la politique des déplacements (SenStadt, 2005). En ce qui concerne les espaces centraux, il s'agit de réduire le trafic de transit, notamment en installant des feux, en réduisant la chaussée ou en élargissant les trottoirs. En outre, les aménagements en faveur des piétons - sécurisation des cheminements - et des cyclistes - extension du réseau cyclable, amélioration du stationnement et de l'intermodalité - se poursuivent, notamment dans l'Est. L'objectif est donc, comme à Paris, le développement de la marche et des " circulations douces ", mais il faut souligner que la politique des déplacements ne s'articule pas aussi fortement sur le réaménagement des espaces publics. Les difficultés financières de la ville (Zawatka-Gerlach,

\footnotetext{
${ }^{12}$ Allié aux Verts, B. Delanoë (PS) emporte les élections contre J. Tibéri, maire RPR entre 1995 et 2001.

${ }^{13}$ En cohérence d'ailleurs avec le Plan des Déplacements Urbains d'lle-de-France (PDUIF) qui définit les principes d'organisation des déplacements et du stationnement à l'échelle régionale.

${ }^{14}$ Le " Mobilien », mesure phare du PDUIF, a pour objectif de constituer un véritable " métro de surface " à partir de 150 lignes de bus (dont 17 à Paris).
} 
2005 ) et des principes d'aménagement en conséquence plus pragmatiques ${ }^{15}$ expliquent en partie cette différence. Il est également vrai que, dans une ville aux densités moindres qu'à Paris, la voirie est suffisamment large pour accueillir tous les usagers sans conflits majeurs.

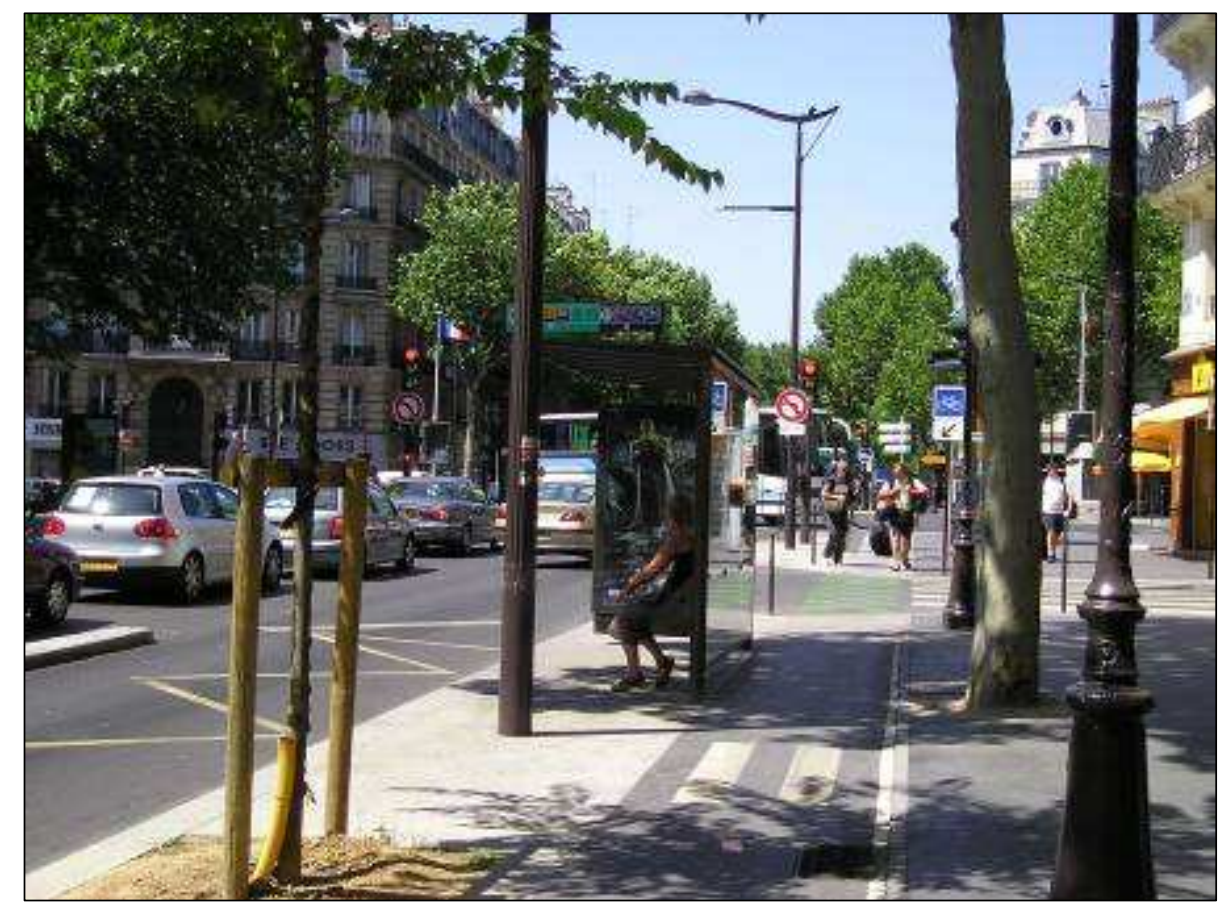

Figure $n^{\circ} 2$. Le boulevard Magenta réaménagé en " espace civilisé » (물 Antoine Fleury, 16/07/2007)

Deux files de circulation au lieu de quatre, création de couloirs de bus et de pistes cyclables, plantation d'une deuxième rangée d'arbres

\section{Améliorer le cadre de vie dans la proximité : un investissement plus ou moins important et des localisations différenciées}

Dans son acception la plus large et la plus partagée, le mot " environnement » (Umfeld) évoque tout ce qui est " autour de nous" (Collomb \& Guérin-Pace, 1999). La question environnementale se confond alors avec la notion de cadre de vie, à une échelle très locale. Longtemps négligé par les pouvoirs publics, le cadre de vie est désormais pris en compte dans tous les projets d'aménagement. Au cœur des métropoles, l'enjeu est de rendre la ville plus agréable à vivre. Pour cela, plusieurs outils sont à la disposition des pouvoirs publics, parmi lesquels l'aménagement des espaces publics tient une bonne place. Ainsi, les aménagements réalisés dans le cadre de la politique des déplacements ne visent pas exclusivement la diminution de la circulation automobile. Leur but est désormais aussi d'améliorer le confort des cheminements piétonniers ou encore la qualité esthétique d'une avenue ou d'une place. Dans un contexte de fortes densités, comme c'est le cas à Paris, l'enjeu est de limiter les nuisances que les autres fonctions urbaines (et les déplacements qu'elles entraînent) font subir aux résidants. A Berlin, il s'agit plutôt de maintenir les

${ }^{15}$ Entretien du 21/04/2005 avec M. Bunge, Direction de la circulation au sein de l'administration du Sénat. Ainsi la mise en place d'un plan de développement de l'espace public (Stadtentwicklungsplan Öffentlicher Raum) a-t-elle été abandonnée. 
habitants dans leur quartier et dans le centre-ville, en rendant ceux-ci plus attractifs, dans un contexte de forte mobilité résidentielle (Häußermann \& Kapphan, 2002).

Variantes berlinoises et parisiennes des "cours urbaines" hollandaises, la "verkehrsberuhigte Zone " (figure $n^{\circ} 1$ ) et le "quartier tranquille » (1995-2001) puis le " quartier vert » (depuis 2001) constituent de nouvelles formes d'aménagement qui ont en commun d'inscrire dans le paysage les limites d'un " quartier ${ }^{16}$, au moyen de matériaux et de revêtements ou d'équipements spécifiques. En fonction de la volonté politique et des financements disponibles, la restructuration des espaces publics à l'intérieur des périmètres est plus ou moins poussée. Le premier objectif est de supprimer la circulation de transit et de diminuer la vitesse, ce qui passe par une modification du plan de circulation, un réaménagement des carrefours et/ou la réduction de la surface des chaussées. Ensuite, il est possible d'aller plus loin dans l'amélioration du cadre de vie, en requalifiant totalement les espaces publics : refonte de l'éclairage, utilisation de matériaux et d'équipements plus nobles, augmentation de la présence végétale ${ }^{17}$. A Paris, ce type de requalification n'est pas rare, même si l'ampleur des aménagements n'est pas toujours aussi importante que le discours politique pourrait le laisser croire, notamment en termes de "végétalisation ». A Berlin, la crise financière limite considérablement l'ampleur des aménagements. Même s'il existe des objectifs en termes d'image et de patrimoine ${ }^{18}$, les pouvoirs publics se concentrent plutôt aujourd'hui sur la dimension fonctionnelle des aménagements (gestion de la circulation, sécurité des usagers) : en dehors des quartiers les plus emblématiques, les espaces publics nouvellement aménagés sont donc moins luxueux que ce qui se fait à Paris.

Les périmètres aménagés selon ces principes doivent recouvrir à moyen terme l'ensemble des espaces centraux. Pour autant, le processus ne se fait que progressivement, donnant à voir des choix qui varient d'une ville à l'autre. Sans négliger les autres types de quartiers, la Mairie de Paris met l'accent d'une part sur les quartiers en voie de transformation, notamment sur le plan socio-démographique, principalement localisés dans l'Est de la capitale (Clerval, 2007), d'autre part sur les quartiers marqués par une grande précarité, notamment dans la couronne parisienne où l'on trouve de nombreux quartiers longtemps délaissés et de surcroît soumis aux nuisances quotidiennes du boulevard périphérique (Fleury, 2007). A Berlin, l'objectif est aussi d'étendre ce modèle d'aménagement à tous les quartiers. Ce sont d'ailleurs les quartiers orientaux de la ville qui sont concernés au premier chef, les quartiers occidentaux ayant été pour la plupart aménagés durant les années 1970 et 1980 . Mais leurs faibles marges de manœuvre poussent les collectivités publiques - les Bezirke (arrondissements) et le Sénat - à investir prioritairement dans les quartiers les plus défavorisés, à l'Ouest dans les quartiers d'habitat social, mais surtout à l'Est dans les quartiers anciens laissés à l'abandon sous le régime communiste.

\footnotetext{
${ }^{16}$ Défini par les pouvoirs publics pour ce seul objectif, ce « quartier » ne coïncide généralement pas avec les autres découpages, mais dépend plutôt de la configuration des rues et des grands axes, ces derniers faisant le plus souvent office de limite.

${ }^{17}$ Ce dernier élément va plus de soi à Berlin, où associations et habitants s'impliquent depuis longtemps dans la plantation et l'entretien des végétaux, même les pouvoirs publics tentent de développer la " végétalisation » à Paris.

${ }^{18}$ Voir le Handbuch zur Gestaltung von Straßen und Plätzen (Manuel pour l'aménagement des rues et des places) rédigé dans les années 1990.
} 


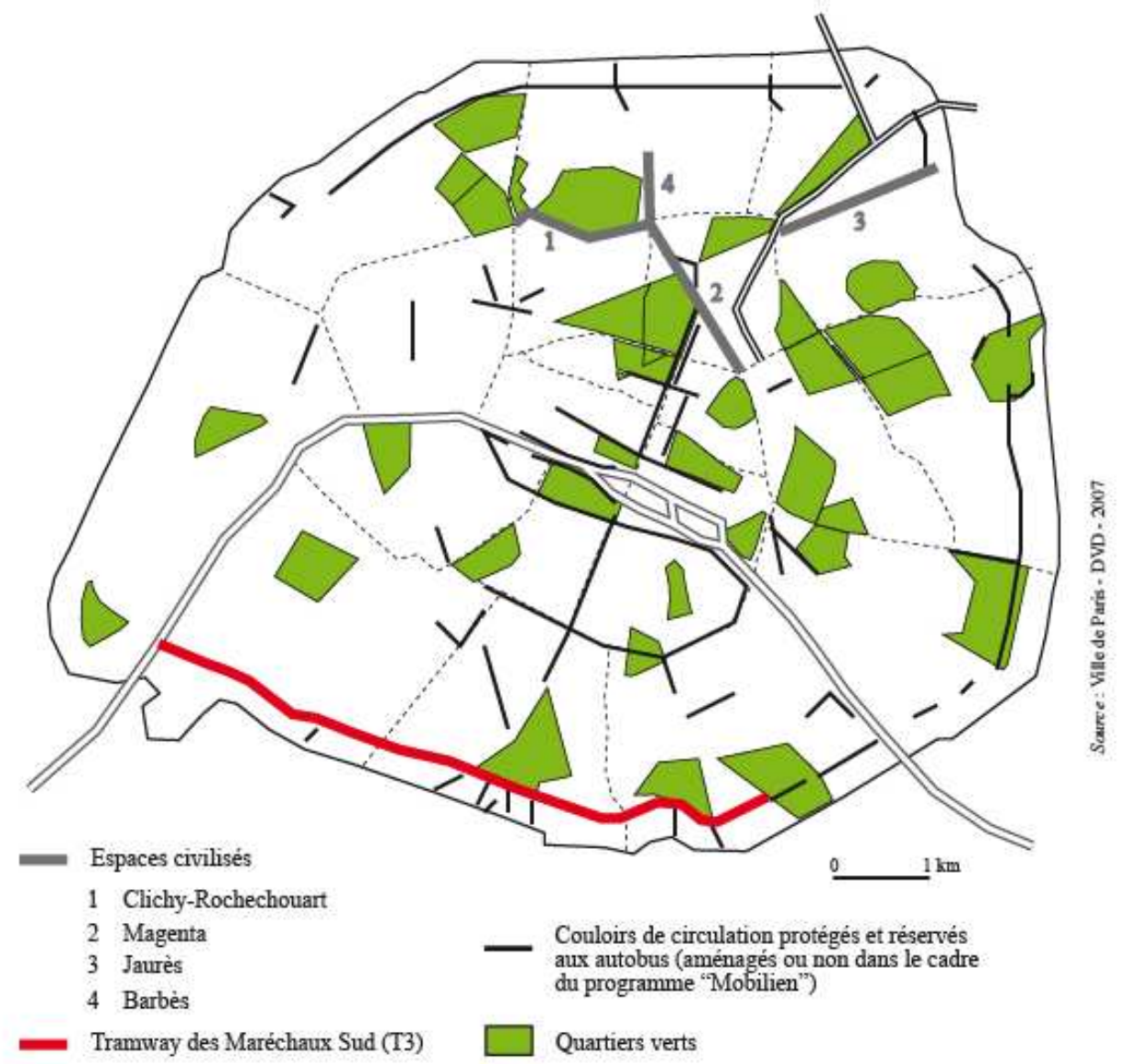

\section{Environnement, espaces publics et jeux d'acteurs}

Si l'on s'intéresse maintenant aux jeux d'acteurs, on constate que la tendance à mettre les espaces publics au service de l'environnement urbain va de pair avec de nouvelles conditions de production. Cette tendance se vérifie à Paris comme à Berlin. Donnant une place considérable à la concertation, les nouveaux modes de production ne font que renforcer la prise en compte de l'environnement.

\section{Espaces publics et concertation locale}

Depuis les années 1970, les pouvoirs publics se sont réorganisés pour être au plus proche des citoyens ${ }^{19}$, ce qui n'est pas sans conséquence sur la production des espaces publics. Le cercle des acteurs impliqués s'est en effet considérablement ouvert sur la société civile. Les associations jouent désormais un grand rôle. Beaucoup d'entre elles militent pour une meilleure intégration de l'environnement dans l'action publique à

${ }^{19}$ Le même mouvement se retrouve dans la plupart des pays d'Europe occidentale. Voir notamment Baquet, Rey \& Sintomer, 2005. 
l'échelon de la ville : associations d'usagers des transports en commun, de cyclistes ou de piétons, associations écologistes. D'autres types d'associations expriment quant à elles des préoccupations plus locales : associations de locataires ou de propriétaires, associations de défense du cadre de vie. Enfin, la parole des habitants est désormais directement prise en compte. Leurs préoccupations en faveur du cadre de vie s'expriment d'ailleurs clairement, que ce soit au moment des campagnes électorales ou, de manière plus fréquente, dans les instances de la démocratie locale.

A Paris, les espaces publics sont l'objet de débats récurrents dans les instances de concertation. La Commission Extra-municipale des déplacements (CEMD) qui, outre un certain nombre d'acteurs publics, regroupe la plupart des associations d'usagers, donne son avis sur les aménagements d'échelle parisienne ("Mobilien » en particulier). Les Comités d'Initiative et de Consultation des Arrondissements (CICA), qui rassemblent acteurs publics et associations, discutent quant à eux des questions d'environnement et de déplacements à l'échelle des quartiers. Si la concertation avec les associations existe depuis les années 1980, les conseils de quartier - qui représentent une évolution importante puisqu'ils impliquent les habitants - n'ont été généralisés qu'après la Loi Démocratie et Proximité de 2002. Ce sont eux qui organisent les débats précédant et accompagnant la mise en œuvre des projets. Initialement consultés pour les aménagements d'échelle locale, ils tendent à être de plus en plus associés aux projets d'échelle parisienne, aux côtés de la CEMD. Plus généralement, l'animation du quartier, le cadre de vie et notamment la voirie, constituent les sujets de discussion les plus récurrents au sein des conseils de quartier ${ }^{20}$. Les espaces publics sont au cœur des préoccupations exprimées par les habitants dans ce cadre $^{21}$ et notamment la présence végétale, la sécurité des piétons aux abords des écoles et dans les rues commerçantes, ou encore la question des nuisances liées à la circulation.

A Berlin, l'implication de la société civile dans les débats publics est ancienne, et les initiatives citoyennes assez courantes. A l'échelle du Land, le Stadtforum (Forum urbain) a permis à des professionnels de la ville, des journalistes et des scientifiques de débattre de l'aménagement de la ville, et notamment de ses espaces publics. A l'échelon local, la concertation est mise en œuvre au quotidien dans les arrondissements ${ }^{22}$, qui sont compétents sur la majorité des espaces publics. Les réunions d'acteurs locaux apparaissent le plus souvent comme un préalable aux aménagements ${ }^{23}$. A la fin des années 1990, le programme Soziale Stadt (ville sociale) met en place le Quartiersmanagement qui renforce encore cette démarche dans certains quartiers (Röcke \& Sintomer, 2003). C'est la société privée ou la fondation en charge de la politique de la ville à l'échelon local qui définit, conformément à son contrat et en concertation avec les habitants, les projets à mettre en

\footnotetext{
${ }^{20}$ Voir la publication annuelle de la Mairie de Paris : Partageons nos expériences pour construire et enrichir la démocratie locale dont l'analyse détaillée pour 2007 montre que 35\% des actions concernent l'aménagement des espaces publics et $25 \%$ l'animation du quartier (qui passe en grande partie par des fêtes organisées dans l'espace public).

${ }^{21}$ C'est du moins ce qui ressort de la consultation des conseils de quartier lors de l'élaboration du PLU : les espaces publics constituent le sujet de $46,9 \%$ des propositions.

${ }^{22}$ Le rôle des arrondissements n'a cessé de se renforcer depuis les années 1980 (Schlusche, 1997). La Constitution de 1990 a pris acte de ce renforcement de l'échelon local en définissant deux collectivités : l'arrondissement, compétent dans tous les domaines de la vie quotidienne, et le Sénat, qui définit les grandes orientations et la planification à l'échelle de l'agglomération.

${ }^{23}$ Entretien avec Mme Ziemer, élue et adjointe au maire de l'arrondissement de Schöneberg-Tempelhof (20/04/2005).
} 
œuvre ; pour cela, elle réunit régulièrement sur place les différents acteurs. Ainsi, bien que le volet social soit initialement prépondérant, c'est selon la volonté des habitants que le travail des premières années a été principalement consacré aux espaces publics ${ }^{24}$, dans un objectif d'amélioration du cadre de vie et des relations de voisinage. Des places, des aires de jeux et des squares ont été créés ou réaménagés; des mesures ont été prises pour améliorer la propreté et la sécurité.

\section{Une réorganisation des pouvoirs publics au service de l'environnement?}

Les acteurs publics sont multiples et s'inscrivent dans différentes structures institutionnelles fonctionnant selon des logiques techniques et fonctionnelles bien définies. Pour les rues et les places, il existe des services de voirie, des services des parcs et jardins, des services de la propreté qui ont des cultures différentes et se composent de corps de métiers divers. Cependant, ces espaces publics ont longtemps été assimilés à l'objet technique qu'est la voirie (Olagnier, 2003), celle-ci étant conçue, aménagée et gérée par une seule direction ${ }^{25}$, les autres directions n'étant sollicitées que pour des aspects bien précis (arbres et jardinières, nettoiement), rarement pour la conception assurée par les ingénieurs de voirie. Mais perçue comme un frein au développement d'une approche globale des espaces publics, cette segmentation s'atténue progressivement. D'importants efforts de coordination sont déployés. La démarche de projet - développée précocement dans des villes comme Barcelone (Olagnier, 2003) ou Lyon (Toussaint \& Zimmermann, 2001) - a notamment été reprise par la Mairie de Paris. Organe administratif naguère chargé de trancher les différends entre les services de la Ville (Spitz, 2004), le Secrétariat Général assure un rôle-pivot dans cette évolution. En organisant régulièrement des rencontres entre les acteurs ou en réfléchissant à une réorganisation des services, il recherche les moyens de rendre l'action plus cohérente et plus efficace.

En même temps, l'environnement tend à devenir l'un des piliers de l'action, et la place des acteurs en charge de cette question se renforce dans le processus de production des espaces publics. Les élus sont pour beaucoup dans cette évolution. A Paris, leur poids

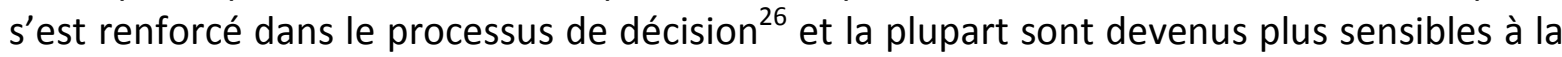
question environnementale ; la présence d'élus " verts » à la tête de certains services clés a aussi largement accéléré le processus ${ }^{27}$. L'un des enjeux est la prise en compte des problèmes d'entretien et de gestion par les concepteurs. La DPE et la DPJEV sont ainsi respectivement amenées à donner leur expertise sur les choix en termes de nivellement et de végétaux. En collaboration avec leur adjoint de tutelle, les deux directions veillent respectivement à ce que la reconfiguration de la voirie prenne en compte les contraintes d'entretien, et que la plantation de végétaux se fasse dans les meilleures conditions

\footnotetext{
${ }^{24}$ Entretiens réalisés avec les responsables des Quartiersmanagements Schöneberg Norden (arrondissement de Schöneberg-Tempelhof) et Helmholtzplatz (arrondissement de Pankow-Prenzlauer Berg) en avril et juin 2007.

${ }^{25}$ Direction de la voirie à Paris ou Tiefbauamt à Berlin (Service des travaux publics).

${ }^{26}$ Depuis le milieu des années 1990, le fonctionnement préfectoral laisse ainsi progressivement place à un fonctionnement plus démocratique (Spitz, 2004).

${ }^{27}$ Entre 2001 et 2007, il s'agit de D. Baupin, adjoint chargé de la voirie et des déplacements, qui a accéléré la mise en œuvre d'une politique de réduction de la circulation automobile, et d'Y. Contassot, adjoint chargé de l'environnement, des espaces verts et de la propreté, qui a promu l'intégration de la logique environnementale dans tous les projets d'aménagement et défendu les concepts de "végétalisation » ou de " corridor écologique ».
} 
possibles pour leur croissance. La DPJEV a même été en partie réorganisée pour accompagner cette évolution. Un service des espaces publics existe d'ailleurs désormais au sein de cette direction, qui travaille en étroite collaboration avec la DVD et la DU. Son action porte sur le renforcement de la présence végétale dans les rues, mais aussi sur les murs - ce qui se traduit par la construction de « murs végétalisés ».

Malgré tout, une partie de l'administration parisienne résiste à cette double transformation des modes de production. Ces résistances au décloisonnement et à la coordination, mais aussi à l'approche environnementale, s'appuient sur de fortes cultures de direction et de corps au sein de la Ville de Paris. Elles s'inscrivent dans un conflit assez courant, mais rendu plus aigu par la taille et l'ancienneté de l'administration parisienne, entre administration et élus, entre la conception que la première se fait de l'intérêt général et les objectifs politiques fixés par les seconds (Spitz, 2004). Cette situation explique le recours à des acteurs extérieurs, pour certains projets : des chargés de mission, au sein de l'administration, ou encore des architectes et des paysagistes appartenant à des cabinets privés, contribuent à faire évoluer les conceptions, notamment en ce qui concerne la qualité environnementale des aménagements d'espaces publics.

Bien que le contexte institutionnel et politique soit différent à Berlin, on y retrouve certains traits de cette évolution. L'environnement est bien intégré dans les politiques publiques, même si la réunification a rendu nécessaire une nouvelle impulsion. Cette question est même relativement consensuelle parmi les acteurs institutionnels, qui ont largement modifié leurs représentations de l'espace public et leurs méthodes de travail. Les services en charge des espaces publics dépendent pour la plupart des Bezirke : si le Sénat conçoit la politique des déplacements à l'échelle du Land et veille à sa mise en œuvre, ce sont les Bezirke qui appliquent cette politique à l'échelon local et qui pilotent les projets d'aménagement visant à améliorer le cadre de vie. La mise en œuvre d'une politique d'aménagement des espaces publics se fait donc principalement à l'échelon local, ce qui facilite une coordination des services qui est d'ailleurs largement entrée dans les mœurs. Pour autant, certains Bezirke ont fait évoluer leur administration pour être encore plus efficaces dans ce domaine : c'est le cas à Mitte où un Straßen- und Grünanlagenamt (Service des rues et des espaces verts) a été créé. La récente réforme des Bezirke, qui conduit à une réorganisation administrative dans un double objectif d'économies et de proximité, n'a pas radicalement modifié la situation, si ce n'est que la baisse des ressources financières rend plus difficile la mise en œuvre des politiques publiques à l'échelon local.

\section{Une approche réductrice des espaces publics ?}

L'intégration des enjeux environnementaux dans la production des espaces publics, aussi bien en ce qui concerne le contenu que les modalités de l'action, pose un certain nombre de questions quant à la nature même des espaces publics. Peut-on parler d'une approche réductrice des espaces publics?

\section{La politique des déplacements : une vision néo-fonctionnelle de l'espace public?}

La plupart des aménagements développés dans le cadre de la politique des déplacements continu finalement de s'inscrire dans une optique fonctionnelle. Ce sont les déplacements qui sont pris en compte plus que l'espace public en lui-même, celui-ci constituant un simple outil au service d'une politique. Les aménagements conduisent à un 
nouveau type de zonage : les voies se scindent en bandes " affectées » à tel ou tel mode de déplacement, ce que certains détracteurs de cette politique appellent à Paris le "laniérage ${ }^{28}$. L'espace public apparaît alors moins comme un tout vécu et pratiqué par des citadins, qui peuvent s'y promener ou y faire des rencontres, que comme un espace traversé au moyen de différents modes de déplacement. L'approche environnementale rencontre ici ses limites, que certains agents et services de la Ville de Paris ne se gardent pas de dénoncer, ce qui explique aussi certaines de leurs résistances aux politiques actuelles.

Pour autant, il faut noter que les pouvoirs publics tendent à corriger cette approche et à mieux prendre en compte la qualité des espaces publics, voire plus globalement leur dimension sociale. Le cas des "espaces civilisés " à Paris en témoigne, et notamment l'aménagement du boulevard Magenta. Comme le confirment la plupart des acteurs interrogés, les pouvoirs publics ont bel et bien atténué l'aspect très fonctionnel des objectifs initiaux - réduction du nombre de files de circulation et création de couloirs réservés pour les bus et les vélos - pour mieux prendre en compte les usages, le patrimoine et la qualité du cadre de vie. Une mission a d'ailleurs été créée au sein du Secrétariat Général, chargée de veiller à la qualité des aménagements. Pour cela, les pouvoirs publics jouent aussi de plus en plus sur les temporalités. C'est le cas avec l'opération "Paris respire ", qui consiste à fermer certaines rues les week-ends et l'été. II n'est d'ailleurs pas rare que ces fermetures soient associées à des fêtes de rue ou à des festivités, comme "Paris Plage ». Plus souples et moins coûteuses que des travaux de voirie, ces opérations permettent elles aussi la réappropriation par les piétons et les « circulations douces » de la rue. Ce type d'action vient enfin de plus en plus compléter les aménagements de voirie eux-mêmes ${ }^{29}$. Ainsi les pouvoirs publics dépassent-ils une approche strictement fonctionnelle pour prendre en compte toute la dimension sociale et culturelle de l'espace public.

Les pouvoirs publics tendent plus généralement à favoriser l'animation des espaces publics au niveau local. Ils s'impliquent ainsi de plus en plus pour que les commerces de proximité se maintiennent dans les quartiers résidentiels, puisqu'ils font une grande partie de la vie de ces quartiers. A Paris, cette préoccupation est inscrite dans le nouveau Plan Local d'Urbanisme (PLU). De plus, la Mairie facilite voire même encourage les habitants, mais surtout les acteurs locaux que sont les associations ou les commerçants, à organiser les animations les plus diverses: marchés spécialisés, repas ou fêtes de quartier, videgreniers, carnavals, etc. C'est une autre manière de faire que les habitants s'attardent dans les espaces publics, qu'ils les pratiquent de manière plus régulière et plus diversifiée. Que ce soit par ce biais ou par celui des aménagements, l'un des objectifs de la Mairie est de favoriser les pratiques de proximité et de recréer du lien social à l'échelle des quartiers: dans ces espaces reconquis sur l'automobile, il faut que les citadins se rencontrent, échangent dans toute leur diversité. Et la question environnementale rejoint la dimension

\footnotetext{
${ }^{28}$ Entretien du 17/11/2004 avec M. Blanchecotte (Directeur du Service Départemental de l'Architecture et du Patrimoine de Paris).

29 Dans le cadre du programme "Mon quartier a de l'avenir », des "rencontres festives " sont ainsi organisées pour " marquer la fin des travaux » et « sensibiliser les riverains au nouveau partage de l'espace public, tout en favorisant les échanges et la convivialité dans les quartiers " (source: www.paris.fr/portail/deplacements).
} 
sociale des espaces publics qui avait présidé à leur émergence comme catégorie de l'action publique.

\section{Produire les espaces publics à l'échelon local : une appropriation par les résidants?}

Nous avons montré ci-dessus que la production des espaces publics se faisait de plus en plus dans le débat qui met en relation les acteurs institutionnels et les habitants ou les associations. Dans les réunions publiques, mais aussi dans ces nouvelles formes de rassemblements actifs que sont, à Paris comme à Berlin, les "marches exploratoires " (où les habitants commentent, critiquent, donnent des idées à leurs élus ou aux techniciens), se constitue un espace public de débat sur l'aménagement des espaces publics. A partir de questions très locales, les habitants sont amenés à prendre position sur de véritables questions de société, à débattre de sujets proprement politiques (Rullier, 2004), et notamment à prendre conscience qu'en tant que portion de sol urbain permettant la mobilité dans la ville, l'espace public est un « bien commun » (Metzger, 2004).

Cependant, on ne saurait se limiter à cette vision somme toute assez idyllique de la concertation et il faut s'interroger sur les intérêts qui se font entendre. A l'échelon local, il faut distinguer un intérêt collectif, exprimé par une majorité des habitants participant à la concertation, et des intérêts particuliers, qui interfèrent et se contredisent mais qui pèsent fortement sur les choix politiques. Les préoccupations collectives renvoient principalement, en tout cas à Paris, à une exigence de protection tous azimuts - notamment face à la circulation - doublée d'une exigence d'embellissement. En ce qui concerne les intérêts particuliers, ils touchent souvent à l'aménagement de couloirs de bus ${ }^{30}$ ou à l'abattage $d^{\prime}$ 'arbres, la plupart du temps en tout cas à la question du stationnement ${ }^{31}$ qui nourrit de nombreuses controverses. Non seulement les projets d'aménagement sont alors tirés vers une dimension locale voire micro-locale en contradiction avec l'intérêt général : réduire l'aménagement d'un espace public à une somme d'intérêts locaux ou particuliers risque de remettre en cause la cohérence paysagère de la ville; les aménagements locaux défavorables à la circulation posent aussi un autre problème fondamental qui est celui de la fluidité, indispensable au bon fonctionnement de la ville et de l'ouverture de ses quartiers aux usagers de passage. Mais au final, les espaces publics sont donc de plus en plus aménagés pour les résidants - les élus cherchant avant tout à rendre visible leur action et à témoigner de leur capacité d'écoute pour se maintenir aux élections suivantes - et par les résidants eux-mêmes, qui sont désormais intégrés aux processus de décision, au détriment des usagers de passage ou de ceux que l'on considère comme "indésirables " (sans-logis, alcooliques, drogués, etc.). Et l'espace public de devenir l'objet des conseils de quartier et des associations de riverains, ceux-ci fonctionnant plus comme les membres d'une copropriété que comme des instances démocratiques.

Enfin, le jeu de la démocratie locale fait que l'on consulte principalement les habitants, et notamment les franges les plus favorisées de la population, qui sont celles qui participent le plus - quantitativement et qualitativement - à la démocratie locale

\footnotetext{
30 L'aménagement des lignes du "Mobilien » dans les $13^{\mathrm{e}}$ et $14^{\mathrm{e}}$ arrondissements en témoigne, avec les nombreuses controverses que celui-ci a suscitées (Fleury, 2007).

${ }^{31}$ Nombreux sont les riverains à ne se déplacer dans les réunions de concertation que pour se plaindre de la suppression de places de stationnement. Les Parisiens sont d'ailleurs très partagés sur cette question, comme en témoigne l'enquête sur le PLU. A la Direction de la voirie, on confirme c'est un "point dur ", une " question récurrente » posée au moment de chaque aménagement.
} 
(Blondiaux, 2004). Ce sont donc les classes moyennes et supérieures qui s'impliquent majoritairement dans la concertation. C'est d'autant plus le cas que ces catégories de population sont aujourd'hui surreprésentées dans les espaces centraux, et qu'elles tendent même à s'y renforcer (Clerval, 2007 ; Häußermann \& Kapphan, 2000). Cela explique en partie les préoccupations évoquées ci-dessus, mais aussi le fait même que les espaces publics aient été érigés comme un thème majeur au sein des structures de la démocratie locale. Les exigences de ce public vont plutôt vers l'amélioration ou la préservation du cadre de vie, que ce soit sur le plan esthétique ou en termes d'usages. C'est aussi dans les contextes où domine ce profil de population que la démocratie locale et plus globalement la vie publique apparaissent les plus actives, que les intérêts locaux s'y expriment d'autant plus. A l'inverse, les franges les plus défavorisées de la population ne prennent que très rarement part à la concertation. La très nette sous-représentation de ce public explique sans doute aussi que des thèmes tels que le logement ou l'action sociale soient peu abordés dans les forums locaux ${ }^{32}$. De plus, la démocratie locale fonctionne souvent moins bien dans les contextes défavorisés. Pour autant, on ne saurait passer sous silence les efforts des pouvoirs publics pour dépasser ce problème. Des dispositifs particuliers permettent à ces habitants de s'impliquer dans la vie publique locale et, plus précisément, dans la production des espaces publics. Les exemples des Grands Projets de Renouvellement urbain (GPRU) et des quartiers de la politique de la ville à Paris, du Quartiersmanagement à Berlin en témoignent. Pour autant, ces dispositifs ne concernent que les quartiers les plus défavorisés, n'englobant pas les quartiers plus mixtes alors que c'est précisément là que se concentre une part importante des aménagements d'espaces publics.

\section{Conclusion}

A l'heure où les enjeux environnementaux sont placés au cœur des politiques publiques, on peut constater que l'aménagement des espaces publics constitue précisément un outil au service de ces nouveaux objectifs. Certes, d'une métropole à l'autre, les aménagements d'espaces publics ont une place variable dans les politiques mises en œuvre, d'autres outils pouvant aussi être mis en avant. Mais ils jouent la plupart du temps un rôle, que ce soit dans le cadre de la politique des déplacements ou dans celui de la revalorisation du cadre de vie. Et ce choix politique va de pair avec la mise en œuvre de nouveaux modes de production marqués par la coordination des acteurs publics et la concertation avec les acteurs locaux. Evidemment ces nouvelles méthodes d'action ne prennent sens que dans le cadre d'une évolution plus profonde des institutions et de la démocratie. Elles ne sont pas liées qu'à la volonté de prendre en compte l'environnement dans l'action publique. Mais ces deux dimensions n'en sont pas moins intrinsèquement liées: d'une part la concertation a été mise en place pour associer les habitants à la transformation du cadre de vie sur lequel ils peuvent désormais agir; d'autre part la réorganisation des acteurs publics va de pair avec la montée en puissance d'acteurs tels que les élus "verts " et plus généralement des services et métiers compétents dans le domaine de l'environnement, qui portent une vision différente des espaces publics.

\footnotetext{
32 La consultation des conseils de quartier au moment du PLU en témoigne pour Paris : 7,5 \% des propositions pour les équipements et seulement $2,1 \%$ pour le logement.
} 
Cependant, mettre les espaces publics au service de l'environnement urbain pose un certain nombre de questions. Tout d'abord, la dimension environnementale des espaces publics ne doit pas faire oublier les autres dimensions de ces espaces. On ne peut pas réduire les espaces publics à un simple outil pour réduire la circulation automobile, ce qui se traduit souvent par le "laniérage » de la voirie. On ne peut pas non plus les considérer exclusivement comme le cadre de vie des résidants, ce que les modes de production actuels tendent à renforcer. En effet, on a pu voir que les simples usagers sont peu représentés dans les instances de débat, au profit d'un rôle exacerbé des résidants. Le risque serait alors de réduire l'espace public à un espace destiné aux seuls habitants du centre, voire à un décor certes de qualité, mais ayant perdu une partie de sa dimension publique d'échanges et de rencontres. II ne faut pas perdre de vue non plus que donner la priorité à des aménagements d'espaces publics, c'est en fait répondre aux exigences des classes moyennes et supérieures essentiellement, et que cela se fait parfois aux dépens d'autres domaines d'action, comme la construction d'équipements sociaux ou de logements. Enfin, ce choix demeure lié aux marges de manœuvres financières de la collectivité en charge des espaces publics, et toutes ne peuvent pas s'investir de la même manière dans cette voie, y compris même en Europe occidentale, comme en témoigne la comparaison entre Paris et Berlin ${ }^{33}$.

\section{Références}

BACQUE M.-H., REY H., SINTOMER Y. (dir.), 2005, Gestion de proximité et démocratie participative. Une perspective comparative, Paris, La Découverte, $320 \mathrm{p}$.

BODENSCHATZ H., 1987, Platz frei für das neue Berlin! Geschichte der Stadterneuerung seit 1871, Berlin, Transit, $285 \mathrm{p}$.

BOURLON A.-M., VILLOT A.-M., 2004, "Espace public et déplacements ", Paris-Projet, n³435, pp. 56-79.

CHALVON-DEMERSAY S. (1984), Le Triangle du XIVe : des nouveaux habitants dans un vieux quartier de Paris, Paris, Édition de la Maison des sciences de l'homme, $176 \mathrm{p}$.

CHERKI E., MEHL D., 1979, Les nouveaux embarras de Paris : de la révolte des usagers des transports aux mouvements de défense de l'environnement, Paris, F. Maspero, $218 \mathrm{p}$.

CLERVAL A. (2007), Evolution de la géographie sociale de Paris, 1982-1999: embourgeoisement et gentrification. Evolution socio-professionnelle de la population des ménages, Paris, APUR, $64 \mathrm{p}$.

COING H. (1966), Rénovation urbaine et changement social, Paris, éditions ouvrières, 295 p. COLLOMB Ph., GUERIN-PACE F., 1998, Les Français et l'environnement: l'Enquête "Populations/Espaces de vie/ Environnements ", Paris, INED/PUF, 255 p.

DECROLY J-M., DESSOUROUX C., VAN CRIEKINGEN M. (2003), "Les dynamiques contemporaines de privatisation des espaces urbains dans les villes européennes ", Belgeo, $\mathrm{n}^{\circ} 1$, pp. 3-19.

DREIF, 2004, Les déplacements des Franciliens en 2001-2002. Enquête Globale Transport. Paris, La Documentation Française.

DUHEM G., GRESILLON B., KOHLER D. (dir.), 2000, Paris-Berlin. Regards croisés sur deux capitales européennes, Paris, Ed. Anthropos, 265 p.

33 L'auteur tient à remercier Thérèse Saint-Julien et Patrick Farges pour leurs relectures avisées, ainsi que le comité de lecture de la revue, dont les observations et critiques ont largement contribué à enrichir cet article. 
DUPUY G., 1995, Les territoires de l'automobile, Paris, Anthropos, 216 p.

FLEURY A., 2007, Les espaces publics dans les politiques métropolitaines. Réflexions au croisement de trois expériences : de Paris aux quartiers centraux de Berlin et Istanbul, Thèse de doctorat en géographie, Université de Paris 1, $665 \mathrm{p}$.

FLONNEAU M., 2001, L'automobile à la conquête de Paris, 1910-1977, Paris, Thèse Université de Paris 1, 3 volumes, 624, $143 \mathrm{p}$.

HAUBOLD D., 1997, Nachhaltige Stadtentwicklung und urbaner öffentlicher Stadtraum, Oldenburg, Bibliotheks- und Informationssystem der Universität, 157 p.

HÄUßERMANN H., KAPPHAN A., 2002, Berlin : von der geteilten zur gespaltenen Stadt? Sozialräumlicher Wandel seit 1990, Opladen, Leske + Budrig, 292 p.

JOYE D., HUISSOUD Th., SCHULER M., 1994, « Le citoyen, la ville et le quartier : participation locale en milieu urbain " in BASSAND M. et al., Les faces cachées de l'urbain, Berne, Peter Lang, pp. 95-111.

LANDAU B., 1993, "La fabrication des rues de Paris au XIX ${ }^{\mathrm{e}}$ siècle, un territoire d'innovation technique et politique ", Annales de la Recherche Urbaine, $n^{\circ}$ 57-58, pp. 24-45.

METZGER P., 2004, "Espace public et bien commun dans l'environnement urbain " in BERDOULAY V. et al., L'espace public à l'épreuve. Régressions et émergences, Pessac, MSHA, pp. 39-48.

OLAGNIER P.-J., 2003, Voirie et espace public : mise en regard de pratiques d'aménagement à Barcelone, Londres et Paris, Thèse de doctorat en géographie, Université de Paris 1, $503 \mathrm{p}$.

RÖCKE A., SINTOMER Y., 2003, " Nouvelles politiques urbaines et démocratie participative en France et en Allemagne ", Grenzgänge 10, n²0, pp. 113-129.

RULLIER B., 2004, "La démocratie à la parisienne », Pouvoirs, n¹10, pp. 9-34.

RYTLEWSKI R., 1999, "Berliner Politik: Zwischen Kiez und Stadtstaat ", in SÜSS W., RYTLEWSKI R., Berlin Die Hauptstadt. Vergangenheit und Zukunft einer europäischen Metropole, Berlin, Nicolai, pp. 295-329.

SCHLUSCHE G., 1997, Die Internationale Bauausstellung Berlin. Eine Bilanz. Planung und Durchführung 1979-1987 und Einfluss und die Berliner Stadtentwicklung, Berlin, Arbeitshefte des Instituts für Stadt- und Regionalplanung, n59, 268 p.

SCHÖBEL S., 2003, Qualitative Freiraumplanung. Perspektiven städtischer Grün- und Freiräume aus Berlin, Berlin wvb, Wiss Verlag, $222 \mathrm{p}$.

SENSTADT, 2005, Berlin in Bewegung. Mobilitätspolitik für das 21. Jahrhundert, Senatsverwaltung für Stadtentwicklung, Berlin, 48 p.

SPITZ P.-E., 2004, "L'administration parisienne, de la noblesse d'Etat à l'entreprise de services ", Pouvoirs, n¹10, pp. 115-128.

TOMAS F., 2002, "L'espace public: un enjeu pour la ville ", in TOMAS F. (dir.), Espaces publics, architectures et urbanité de part et d'autre de l'Atlantique, Publications de I'Université de Saint-Etienne, pp. 13-21.

TOUSSAINT J.-Y., ZIMMERMANN M., 2001, "L'espace public comme pratique d'aménagement ", in TOUSSAINT J.-Y., ZIMMERMANN M., User, observer, programmer et fabriquer l'espace public, Lausanne, PPUR, pp. 5-15.

WERNER M., ZIMMERMANN B., 2004, De la comparaison à l'histoire croisée, Paris, Seuil, $239 \mathrm{p}$.

ZAWATKA-GERLACH U., 2005, Die öffentlichen Finanzen des Landes Berlin. Grundzüge, Prinzipien, Finanzkrise, Berlin, Landeszentrale für politische Bildungsarbeit, 73 p. 